\title{
ON THE STABILITY OF IDENTIFICATION PATTERNS FOR DIRICHLET REGIONS
}

\author{
MARJATTA NÄÄTÄNEN
}

\section{Introduction}

Let $G$ be a purely hyperbolic Fuchsian group of the first kind with genus $g \geqq 1$ and let $D(z)$ be the Dirichlet region for $G$ with center $z$. It was proved by Beardon [2, Theorem 9.4.5] that for a.e. $z D(z)$ has all cycles of length 3 and, hence, $12 g-6$ sides. Let $p(g)$ give the number of possible identification patterns. In [3] we showed that $p(2)=8$. For $i=1, \ldots, p(g)$ let

$$
D_{i}=\{z \mid D(z) \text { has } 12 g-6 \text { sides and pattern } i\} .
$$

It is obvious that the sets $D_{i}$ are invariant under $G$. Here it is shown that the sets $D_{i}$ are open and properties for their boundary curves are proved. Chapters 3 and 4 deal with the case $g=2$. It is shown that the group of the regular octagon, introduced in [4, Theorem 5.1], only has pattern 6 and its degenerates as patterns for $D(z)$. Groups which change their pattern according to elementary moves [3, Chapter 5] are constructed in Chapter 4.

I am indebted to S. Mustonen, I. Mellin and H. Haario for help with computer work, to S. Rokka for the figures, and to M. Lehtinen for valuable comments.

\section{The sets $D_{i}$ and $\partial D_{i}$}

In [2, Theorem 9.4.5] it is shown that if $D(z)$ has less than $12 g-6$ sides, then there exist distinct $f_{i} \in G \backslash I, i=1,2,3$, such that

$$
\operatorname{Im}\left[z, f_{1}(z), f_{2}(z), f_{3}(z)\right]=0,
$$

where the cross-ratio cannot be constant. Since $G$ has a countable number of transformations, the "exceptional" centers lie on a countable number of curves defined by (2.1). Each curve is algebraic, since (2.1) gives a polynomial of degree 8 in two real variables.

Definition 2.1. Let $f_{i} \in G \backslash I$ be distinct, $i=1,2,3$, and let $\gamma$ be the algebraic curve corresponding to the condition (2.1). We define $\Gamma$ as the set of all curves $\gamma$. 
Remark 2.1. By the invariance of the cross-ratio

$$
\left\{z \mid\left[z, f_{1}(z), f_{2}(z), f_{3}(z)\right] \in \boldsymbol{R}\right\}=\left\{z \mid\left[h(z), h f_{1}(z), h f_{2}(z), h f_{3}(z)\right] \in \boldsymbol{R}\right\}
$$

for each Möbius transformation $h$. Hence the set $\Gamma$ could also be defined by replacing conditions of type (2.1) by conditions

$$
\operatorname{Im}\left[g_{1}(z), g_{2}(z), g_{3}(z), g_{4}(z)\right]=0,
$$

where $g_{i} \in G$ are distinct, $i=1,2,3,4$.

Theorem 2.1. Each cycle of length 4 in $D(z)$ corresponds to $z$ lying on a curve $\gamma \in \Gamma$.

Proof. Let $P$ be a vertex of $D(z)$ with the other vertices in its cycle $g_{1}(P), g_{2} g_{1}(P), g_{3} g_{2} g_{1}(P)$. Then $z, g_{1}^{-1}(z),\left(g_{2} g_{1}\right)^{-1}(z)$ and $\left(g_{3} g_{2} g_{1}\right)^{-1}(z)$ are concyclic with center $P$. Because $g_{1}$ is a Möbius transformation, this is equivalent to the points $g_{1}(z), z, g_{2}^{-1}(z)$ and $\left(g_{3} g_{2}\right)^{-1}(z)$ being concyclic with center $g_{1}(P)$. The algebraic curves given by

and

$$
\operatorname{Im}\left[z, g_{1}^{-1}(z),\left(g_{2} g_{1}\right)^{-1}(z),\left(g_{3} g_{2} g_{1}\right)^{-1}(z)\right]=0
$$

$$
\operatorname{Im}\left[z, g_{2}^{-1}(z),\left(g_{3} g_{2}\right)^{-1}(z), g_{1}(z)\right]=0
$$

are the same. A similar argument applies to the other vertices in the cycle of $P$.

Theorem 2.2. If $D(z)$ has one cycle of length 4 and the others of length 3 , then in a neighbourhood $U$ of $z, \bigcup_{i=1}^{p(g)} \partial D_{i} \cap U=U \cap \gamma$, where $\gamma \in \Gamma$, and for $w \in U \cap \gamma$ the identification pattern of $D(w)$ is the same as that of $D(z)$, while when $w$ crosses $\gamma$ in $U \backslash\{z\}$ a side-pairing transformation is changed in $D(w)$.

Proof. If $P$ is a vertex with cycle length 3 , then by continuity and discreteness $z$ has a neighbourhood in which $D(w)$ has a vertex in a neighbourhood of $P$ with the same cycle length and adjacent sides labelled by the same mappings as $P$ has for $D(z)$.

The condition for $Q$ being a vertex of $D(z)$ with cycle length 4 and with adjacent sides labelled by $f_{1}, f_{3}$, the side $f_{2}$ being degenerate at $Q$, is, in hyperbolic distances,

$$
\varrho(Q, z)=\varrho\left(Q, f_{1}(z)\right)=\varrho\left(Q, f_{2}(z)\right)=\varrho\left(Q, f_{3}(z)\right)<\varrho(Q, g(z))
$$

for all $g \in G \backslash\left\{I, f_{1}, f_{2}, f_{3}\right\}$ and $f_{2}(z)$ lies on the circle with center $Q$ on the part between $f_{1}(z)$ and $f_{3}(z)$ not containing $z$. Then also the side $f_{1}^{-1} f_{3}$ is degenerate. Because of continuity and discreteness there exists a neightourhood $U$ of $z$ such that for $w \in U \cap \gamma$, where $\gamma$ is given by (2.1), the pattern of $D(w)$ is the same as that of $D(z)$, while for $w \in U \backslash \gamma, D(w)$ has $12 g-6$ sides since all cycles are of length 3 .

We claim the side-pairing mapping added to $D(z)$ is changed between $f_{2}$ and $f_{1}^{-1} f_{3}$ as the curve $\gamma$ is crossed in $U \backslash\{z\}$. Denote $R(w)=\left[w, f_{1}(w), f_{2}(w), f_{3}(w)\right]$. Then $R$ is not a constant. Let $g_{w}$ be the Möbius transformation which maps $w$, $f_{1}(w), f_{3}(w)$ to $0,1, \infty$, respectively. Then $R(w)=g_{w}\left(f_{2}(w)\right)$. Let $C(w)$ be the open 
disc with $w, f_{1}(w), f_{3}(w)$ on $\partial C(w)$. Then $g_{w}(\partial C(w))=\boldsymbol{R}$ and $g_{w}(C(w))$ is either the upper or lower half-plane. Assume it is the upper one. Then, if $\operatorname{Im} R(w)>0$, it follows that $f_{2}(w) \in C(w)$ and the side labelled by $f_{2}$ appears in $D(w)$ between the sides labelled by $f_{1}$ and $f_{3}$. If $\operatorname{Im} R(w)<0$, then $f_{2}(w)$ is not in the closure of $C(w)$ and there is no side between the sides $f_{1}$ and $f_{3}$ in $D(w)$. Since $R$ is a rational function, there is a neighbourhood $N$ of $z$ such that $R(N)$ covers a neighbourhood of $R(z)$ at least once and for each $w \in N \backslash\{z\} R$ is a homeomorphism when restricted to a neighbourhood $N^{\prime}$ of $w$. If $w \in \gamma \backslash\{z\}$, then $R(w) \in \boldsymbol{R}$.

Denote $U_{1}=\left\{w \in N^{\prime} \mid \operatorname{Im} R(w)>0\right\}, \quad U_{2}=\left\{w \in N^{\prime} \mid \operatorname{Im} R(w)<0\right\} . \quad$ Then, when $w \in U_{1}, D(w)$ has a side labelled by $f_{2}$ and, when $w \in U_{2}, f_{2}$ is not in $D(w)$, and the added side is labelled by $f_{1}^{-1} f_{3}$. The common boundary of $U_{1}$ and $U_{2}$ in $N^{\prime}$ is $\gamma$, hence when $w$ crosses $\gamma$ a side-pairing mapping is changed in $D(w)$.

Theorem 2.3. Let $z \in \bigcup_{i=1}^{p(g)} \partial D_{i}$ and let $l$ be the number of the cycles in $D(z)$ having length $n_{k} \geqq 4, k=1, \ldots, l$. Then in a neighbourhood $U$ of $z, \bigcup_{i=1}^{p(g)} \partial D_{i}$ lies on at most $\sum_{k=1}^{l}\left(\begin{array}{c}n_{k} \\ 4\end{array}\right)$ curves $\gamma \in \Gamma$. For each $\gamma, \gamma \cap U$ is the union of finitely many arcs which intersect only at $z$, each homeomorphic to an open interval of real numbers.

Proof. For $k=1, \ldots, l$ let $P_{k}$ te a vertex of $D(z)$ with length of the cycle $n_{k} \geqq 4$. Then there are exactly $n_{k}$ points of the set $G(z)$ on a circle with center $P_{k}$. Each choice of 4 of them determines a curve $\gamma$ through $z$ by the condition

$$
\operatorname{Im}\left[g_{1}(z), g_{2}(z), g_{3}(z), g_{4}(z)\right]=0 .
$$

As in Theorem 2.1 , by mapping $P_{k}$ to another veriex in the cycle, we see that each vertex in the same cycle gives rise to the same curves $\gamma \in \Gamma$.

Each curve $\gamma$ is the preimage of $\boldsymbol{R}$ under the non-constant rational mapping $R$ of Theorem 2.2. Hence the last assertion holds in a suitably chosen neighbourhood $U$ of $z \in \gamma$.

Remark 2.2. Let $\mathrm{Cl} D(0)$ denote the closure of $D(0)$. It suffices to consider points in $\mathrm{Cl} D(0)$ to investigate the sets $D_{i}, i=1, \ldots, p(g)$, for a group $G$, since the sets $D_{i}$ are invariant under $G$. By Theorem 2.3 and the compactness of $\mathrm{Cl} D(0)$, $\bigcup_{i=1}^{p(g)} \partial D_{i} \cap \mathrm{Cl} D(0)$ is on a finite number of curves $\gamma \in \Gamma$.

Theorem 2.4. Let the group $G$ act on the open unit disc $U$, let $z \in D_{i}$, $i=1, \ldots, p(g)$, and let $B$ be the union of subsets of $U$ fulfilling conditions of type (2.1), where $f_{1}, f_{2}, f_{3}$ label adjacent sides of $D(z)$. Let $W$ be the component of $U \backslash B$ containing $z$. Then $W \subset D_{i}$ and $\partial W \subset \partial D_{i}$.

Proof. Since $z \in D_{i}, \quad i=1, \ldots, p(g), D(z)$ has a maximal number of sides. Hence, because of continuity, for $w \in W, D(w)$ has the same pattern as $D(z)$, since the pattern of $D(w)$ can change from that of $D(z)$ only if some of the sides of $D(z)$ 
degenerate to vertices, i.e., if the center $w$ fulfils at least one condition of type (2.1) for mappings labelling adjacent sides of $D(z)$. Hence $W \subset D_{i}$.

For $w \in \partial W$, a condition of type (2.1) is valid for mappings labelling three adjacent sides of $D(z)$ and, hence, at least one side-pair of $D(z)$ is degenerate in $D(w)$. Thus $\partial W \subset \partial D_{i}$.

Corollary 2.1. The set $D_{i}, i=1, \ldots, p(g)$, is open.

\section{Application to the group of the regular octagon}

In [4] we studied the group with $D(0)$ the regular octagon with diametrically opposite pairings. We now derive the curves $\gamma \in \Gamma$ of Theorem 2.3 through 0 . In this case $\bigcup_{i=1}^{8} \partial D_{i}$ lies on at most $\left(\begin{array}{l}8 \\ 4\end{array}\right)=70$ curves in a neighbourhood of 0 . In Theorem 3.1, the dependence of $D(z)$ on $z$ is studied for this group.

The generators are $f_{1}, f_{2}, f_{3}, f_{4}$ with the relation $f_{1} f_{2} f_{3} f_{4} f_{1}^{-1} f_{2}^{-1} f_{3}^{-1} f_{4}^{-1}=I$ and $f_{k+1}=g^{-k} f_{1} g^{k}, k=1,2,3$, where $g(z)=(\exp (i 5 \pi / 4)) z$.

The cycle of a vertex of $D(0)$ has length 8 and $\bigcup_{i=1}^{8} \partial D_{i}$ is in a neighbourhood of 0 on the curves $\gamma$ obtained from mappings $I, f_{1}, f_{1} f_{2}, f_{1} f_{2} f_{3}, f_{1} f_{2} f_{3} f_{4}, f_{4} f_{3} f_{2}$, $f_{4} f_{3}, f_{4}$, labelling adjacent Dirichlet regions. Since the mappings are Möbius transformations and the generators are conjugates, we obtain for example: if we denote $w=g^{-1}(z)$, then $\operatorname{Im}\left[z, f_{1}(z), f_{1} f_{2}(z), f_{1} f_{2} f_{3}(z)\right]=0$ is equivalent to $\operatorname{Im}\left[f_{1}(w)\right.$, $\left.f_{1} f_{2}(w), f_{1} f_{2} f_{3}(w), f_{1} f_{2} f_{3} f_{4}(w)\right]=0$. Hence the set of points satisfying the latter equation is obtained by rotating by $g^{-1}$ the set of points satisfying the former equation. Hence it suffices to consider the cyclic choices of four of the mappings labelling the Dirichlet regions around $P$ and to take rotations by the powers of $g$ of the algebraic curves we have obtained to get all curves through 0 on which $\bigcup_{i=1}^{8} \partial D_{i}$ can lie in a neighbourhood of 0 . These are shown in Figure 1, calculated by a computer.

Theorem 3.1. The group of the regular octagon only has pattern 6 [3] and its degenerates as patterns for $D(z)$.

Proof. Step 1. To get the patterns for $D(z), z \in \mathrm{Cl} D(0)$, it suffices to consider the sector with angle $[\pi, 5 \pi / 4]$ in the origin: if we denote $f_{i+4}=f_{i}^{-1}, i=1, \ldots, 4$, then $f_{i}=g^{-k} f_{j} g^{k}$ holds for $i, j, k=1, \ldots, 8, j+k=i(\bmod 8)$. Choose $i=1, \ldots, 7$. We claim that the pattern for $D\left(z^{\prime}\right)$, where $z^{\prime}=g^{i}(z)$, is obtained from $D(z)$ by replacing the generator $f_{j}, j=1, \ldots, 4$, by $f_{j-i}(\bmod 8)$. This follows from the fact that $g^{i}$ is a Möbius transformation and $g^{i} f_{j}(z)=g^{i} f_{j} g^{-i}\left(z^{\prime}\right)=f_{j-i}\left(z^{\prime}\right)$.

Step 2. Let $r$ and $R$ be the radii of the inscribed and circumscribed circles for $D(0)$. We claim it suffices to consider $z$ in the triangle $T$ (Figure 2) with vertices in 
the hyperbolic polar coordinates $0, Q=(r, \pi), \quad S=(r, 5 \pi / 4)$. Let $P=(R, 9 \pi / 8)$. The set $D(P)$ is a regular octagon with diagonal pairings by $f_{4} f_{3} f_{2}, f_{1} f_{2} f_{3}, f_{1} f_{2} f_{4}^{-1}$, $f_{1} f_{3}^{-1} f_{4}^{-1}$. Let $h$ be the reflection in the line through $Q$ and $S$. Then $h$ maps $D(P)$ to $D(0)$ and $h^{-1} f_{1} h=\left(f_{4} f_{3} f_{2}\right)^{-1}, h^{-1} f_{2} h=f_{4} f_{3} f_{1}^{-1}, h^{-1} f_{3} h=f_{1} f_{2} f_{4}^{-1}, h^{-1} f_{4} h=\left(f_{1} f_{2} f_{3}\right)^{-1}$. We deduce that $D(h(z))$ is obtained from $D(z)$ by replacing each $f_{i}, i=1, \ldots, 4$, by its conjugate (and reversing the order). Also, the reflections of the curves in $T$ indicating exceptional centers are curves indicating exceptional centers in $h(T)$ belonging to the conjugate mappings.

Step 3. We finish by calculating $D(z)$ for $z \in T$. By continuity, in a neighbourhood of $0, D(z)$ only has sides labelled by $g$, where $D(g(0))$ is adjacent to $D(0)$. We want to find a point $z_{0} \neq 0$ in this neighbourhood and calculate $D\left(z_{0}\right)$.

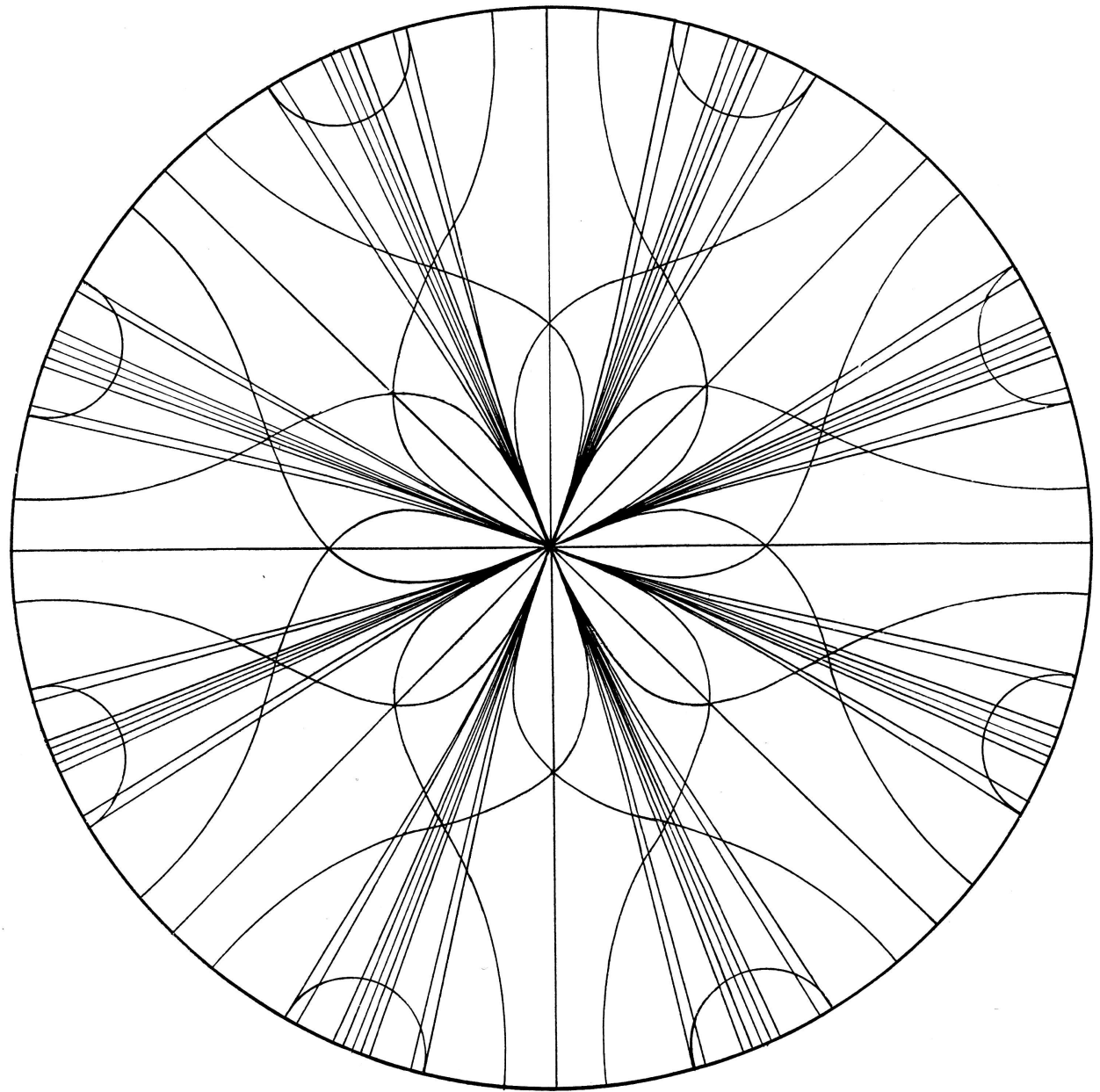

Figure 1 
The set $D(z)$ is in a neighbourhood of 0 obtained from $D(0)$ so that some of the sides degenerated to points open up. The obtained pattern can only change if some of the sides of $D(z)$ degenerate to points. Let $g_{1}, g_{2}, g_{3}$ be adjacent sides of $D(z)$. The side $g_{2}$ degenerates to a point on the curve $\operatorname{Im}\left[z, g_{1}(z), g_{2}(z), g_{3}(z)\right]=0$. There are two cases to consider depending on whether $g_{2}$ labels a side or a "degenerate side" of $D(0)$.

Case 1. The regions $D\left(g_{1}(0)\right), D\left(g_{2}(0)\right), D(0)$ and, respectively, $D\left(g_{2}(0)\right)$, $D\left(g_{3}(0)\right), D(0)$ have a common vertex, the vertices are adjacent, and $g_{2}$ labels the side of $D(0)$ between them. The corresponding curves are obtained by rotation by powers of $g$ from the following special case: Take $f_{1}$ for $g_{2}$. There are six choices for $g_{1}$ and six for $g_{3}$. The number of curves to be drawn by a computer can be diminished by means of symmetry. All obtained curves have euclidean distance at least 0.4 from 0 .

Case 2. The regions $D\left(g_{1}(0)\right), D\left(g_{2}(0)\right), D\left(g_{3}(0)\right)$ and $D(0)$ have a common vertex. The corresponding curves are in Figure 1.

Hence for $D(-0.3,-0.04)$ it suffices to consider the mappings labelling Dirichlet regions adjacent to $D(0)$; it will have 18 sides. The sides are: $f_{1}, f_{1} f_{4}^{-1}$, $\left(f_{4} f_{3} f_{2}\right)^{-1}, f_{2}^{-1},\left(f_{1} f_{2}\right)^{-1}, f_{3}, f_{4}^{-1}, f_{1}^{-1}, f_{2},\left(f_{4} f_{3}\right)^{-1}, f_{3}^{-1},\left(f_{1} f_{2} f_{3}\right)^{-1}, f_{4} f_{1}^{-1}, f_{4}$, $f_{4} f_{3}, f_{4} f_{3} f_{2}, f_{1} f_{2} f_{3}, f_{1} f_{2}$. The pattern is 6 . A side, say $f_{1}$, disappears by becoming a vertex in $D(z)$ if $\operatorname{Im}\left[z, f_{1} f_{2}(z), f_{1}(z), f_{1} f_{4}^{-1}(z)\right]=0$. The curves for all sides are already calculated and the result is that the pattern above is preserved in $T$, with the sides $f_{4} f_{3}, f_{1} f_{2} f_{3}$ degenerating on $0 Q, f_{1} f_{2}, f_{4} f_{3} f_{2}$ on $0 S, f_{2}, f_{3}$ on $Q S$ and $f_{1} f_{4}^{-1}$ at $0, f_{1}$ at $S, f_{4}$ at $Q$.

The conclusion is that the group of the regular octagon has for patterns for $D(z), \quad z \in \mathrm{Cl} D(0)$, pattern 6 , its degenerates with 14 sides on the lines drawn in Figure 2 (and their rotations by powers of $g$ ), and the regular octagon with diagonal identifications at 0 , at the vertices of $D(0)$ ard at the midpoints of sides of $D(0)$. The patterns for $z, z \in \mathrm{Cl} D(f(0))$, where $f$ is in the group, are obtained by conjugation.

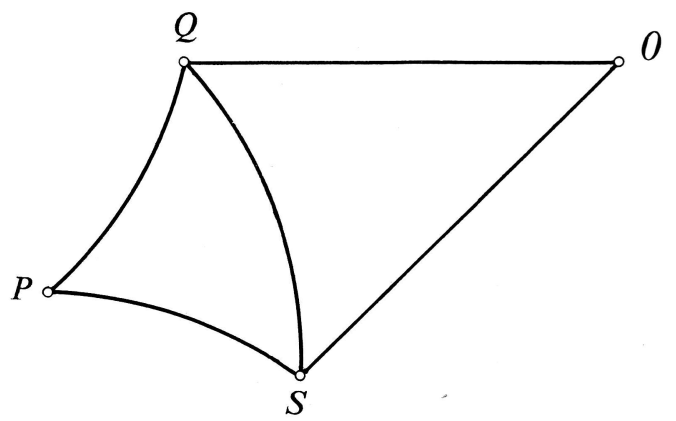

Figure 2 
Remark 3.1. Figure 2 is obtained by using a computer, but exact calculations can also be made. For example, the cross-ratio $\left[z, f_{4}(z), f_{4} f_{2}(z), f_{4} f_{3} f_{2}(z)\right]$ can be represented as a quotient of two polynomials of degree four in $z$, with real coefficients. Hence the side $f_{4} f_{3}$ degenerates on the real axis.

\title{
4. Examples of groups with at least two patterns
}

We construct a group which has points in $D_{1}$ and $D_{2}$. Other groups corresponding to the transition pattern [3, Chapter 5] can be constructed in a similar way.

The group is obtained by constructing $D(0)$ : Let $P$ be a sixteengon with every fourth vertex at an equal distance from 0 and with angles $\pi / 2$, all others at an equal distance from 0 and with angles $2 \pi / 3$, constructed by using [1, Theorem 1]. Then the angle bisectors are concurrent at 0 and the sides of $P$ can be identified by using the third sequence in [3, Chapter 6]. Because of the conditions used, $P$ is $D(0)$ for the group generated by its side-pairing transformations.

The point 0 is on the curve $\gamma \in \Gamma$ where the cross-ratio corresponding to the cycle of length 4 is real, and it follows from Theorem 2.2 that there exists a neighbourhood $N$ of 0 such that in each component of $N \backslash \gamma$ one of the degenerated sidepairs of $D(0)$ opens up and the pair opening up is changed when crossing $\gamma$. The change of the sides changes the pattern between 1 and 2 .

\section{References}

[1] Beardon, A. F.: Hyperbolic polygons and Fuchsian groups. - J. London Math. Soc. (2) 20, 1979, 247-254.

[2] BeARdon, A. F.: The geometry of discrete groups. - Graduate Texts in Mathematics 91. SpringerVerlag, New York, 1983.

[3] JøRGENSEN, T., and M. NÄÄTÄNEN: Surfaces of genus 2: Generic fundamental polygons. - Quart. J. Math. Oxford Ser. (2) 33, 1982, 451-461.

[4] NÄÄTÄNEN, M.: Regular $n$-gons and Fuchsian groups. - Ann. Acad. Sci. Fenn. Ser. A I Math. 7, 1982, 291-300.

\author{
University of Helsinki \\ Department of Mathematics \\ SF-00100 Helsinki \\ Finland
}

Received 19 March 1984 